\title{
Investigations of adult neuroplasticity as an effect of long-term physical activity in old age
}

\author{
Mandy Bade', Anita Hökelmann', Jörn Kaufmann', Volkmar Leßmann', \\ Patrick Müller', Kathrin Rehfeld', Notger Müller² \\ 1 Otto von Guericke University Magdeburg, Magdeburg, Germany \\ ${ }^{2}$ German Center for Neurodegenerative Diseases in Magdeburg, Magdeburg, Germany \\ mandy.bade@med.ovgu.de; anita.hoekelmann@ovgu.de; joern.kaufmann@med.ovgu.de;
} volkmar.lessmann@med.ovgu.de; patrick.mueller@dzne.de; kathrin.rehfeld@ovgu.de; notger.mueller@dzne.de

\begin{abstract}
Introduction: The growing number of elderly people in the population is accompanied by an increased prevalence of chronic diseases, e.g. dementia, which will confront the healthcare system with major challenges in the future. Therefore, appropriate interventions are needed to support healthy aging combined with a high quality of life. The potential of the adult organism for neuronal plasticity, induced by longterm physical activity, was examined in an interdisciplinary project on aging research from neuroscientific, physiological, neuropsychological and sports science perspectives. Methods: In a longitudinal study with 20 healthy elderly people $(\mathrm{M}=72.65, \mathrm{SD}=4.31$ years $)$ the influence of endurance and strength training as well as dance training on brain structure, BDNF, memory, balance ability and endurance performance was investigated. Measurements were performed before the start of the training phase, after 6 months, 18 months and 5 years. Structural MRI scans (FSL segmentation), blood analyses, the Verbal Learning and Memory Test (VLMT), Limits of Stability Test (LoS) and Physical Working Capacity Test 130 (PWC 130) were conducted. Results: In both groups, we observed an increased volume in the left amygdala $(\mathrm{F}(3,36)$ $=3.760, \mathrm{p}=.019, \mathrm{f}=.56)$, a maintenance of BDNF concentration in blood plasma, an improvement in balance ability according to LoS in terms of reaction time $(\mathrm{F}(3,42)=6.379, \mathrm{p}=.001, \mathrm{f}=.67)$, movement velocity $(\mathrm{F}(3,42)=4.925, \mathrm{p}=.005, \mathrm{f}=.59)$, endpoint $(\mathrm{F}(1.83,25.58)=9.221$, $\mathrm{p}=.001, \mathrm{f}=.81)$, maximum dislocation $(\mathrm{F}(3,42)=12.476, \mathrm{p}<.001, \mathrm{f}=$ $.94)$ and direction control $(\mathrm{F}(3,42)=6.542, \mathrm{p}=.001, \mathrm{f}=.68)$ as well as a stabilization of the performance in PWC 130. Memory performance improved significantly in the dance group with regard to recognition performance $\left(\mathrm{X}^{2}(3, \mathrm{~N}=8)=12.197, \mathrm{p}=.007\right)$. Discussion and conclusions: The neuroplasticity effect was confirmed as a result of both training measures based on neurostructural, molecular, cognitive, coordinative
\end{abstract}


and conditional adaptation phenomena in the adult organism. The study showed that a long-term physically active lifestyle leads to preservation of performance and thus to a higher quality of life as well as autonomy in old age. It should be emphasized that only dance training contributed to cognitive performance enhancement.

Keywords: neuroplasticity, cognition, dance, seniors, dementia prevention

\section{Introduction}

Demographic change, characterized by low birth rates and simultaneously rising life expectancy, has arrived in Germany long ago. "Every second person in Germany today is older than 45 and every fifth person is older than 66" (Federal Statistical Office, 2020). In the future, a further increase in the number of senior citizens and a decline in the working-age population can be expected. The challenges for the health care system and the provision of care require the development of appropriate interventions that support healthy aging combined with a high quality of life and autonomy in old age. Physical activity and cognitive training are interventions that have been shown in the past to be promising methods to positively influence age-related structural and functional brain changes, and thus the prevalence of dementia. A combination of both methods seems to be the most effective. Sportive dance training represents such a combination, as it combines conditional, coordinative, cognitive and emotional contents (Müller et al., 2017, Rehfeld et al., 2017, Rehfeld et al., 2018).

Our aim was to investigate the influence of different physical activities on neurostructural, molecular, neuropsychological and motor parameters in healthy elderly people aged 60 to 85 years in the long-term course. A distinction is made between sportive dance training and fitness training (endurance and strength training). The longitudinal study is one of the few to examine the long-term effects of different training methods on the complex performance structure of healthy elderly people and therefore plays an essential role in the research focus on dementia prevention.

\section{Methods}

The longitudinal investigation is based on a randomized study design. In 2012, study participants were randomly assigned to either an intervention group (sportive dance training) or an active control group (fitness training). After six months of intervention, the first posttest was conducted. After another 12 months of training, posttest 2 was performed. Finally, a last posttest was completed about 5 years after the start of the first tests. Over the course of the 5-year longitudinal study, the sample size decreased from the original 62 subjects to 20 subjects (ten subjects per group). The measurements included neurostructural, molecular, neuropsychological and motor tests. The aim of the longitudinal study was to examine the development of the above-mentioned parame- 
ters of the sportive dance group and the active control group in the long-term course of about 5 years. An inactive control group was not included because numerous studies have already shown that physical inactivity leads to accelerated age-correlated decline processes (Erickson et al., 2011). In addition, it is ethically questionable to encourage people to be physically inactive for a longer period.

Only cognitively healthy subjects were included in the study. Inclusion criteria were a minimum score of 26 on the Mini-Mental Status Test (MMST) of Folstein, Folstein \& McHugh (1990). Further inclusion criteria were a minimum age of 60 years and MRI suitability (no metallic implants, pacemakers, tinnitus, tattoos, use of centrally acting drugs, claustrophobia and neurological diseases such as stroke or Parkinson's disease).

In the intervention group, the training of conditional and, in particular, coordinative abilities were in focus, as well as the memorization and recall of acyclic, non-automated movement combinations. The fitness training, on the other hand, emphasized conditional skills such as strength and endurance, which were characterized by cyclic, automated, and alternating movements (Rehfeld, 2016). Both exercise interventions had the same conditions regarding the use of music as well as the implementation of the training in a group to keep possible psychosocial and emotional effects constant in the two groups. The training duration, intensity and frequency were almost identical in both training groups. The structure of the training sessions in a warm-up, main and cooldown part were also the same in both groups. The sportive dance training included different genres, such as line dance, jazz dance, Latin dance, rock'n'roll and square dance, which changed monthly. The training concept was based on a balance between learning new acyclic movement patterns and consolidating them through repetition and recall. Due to the variety of coordination of partial body movements, new multimodal sensory and motor stimuli were created continuously. The combinations of mostly unknown step patterns and arm movements had to be memorized, recalled and reproduced under the pressure condition of the dynamic-rhythmic structure of time. The memorization of movement sequences and the recall of these to specific musical structures under time pressure and the permanent relearning of movement sequences under constant conditional stress underline the high cognitive demand in sportive dance training. In addition to time pressure, rhythm, positioning and precision pressure are further pressure conditions of dancing. Spatial orientation, balance (change of one-legged stances, permanent shifting of the body's center of gravity), directional ability (due to changes of place and direction as well as turns), coupling ability (through the combination of arm and leg movements) and speed were also trained. In the fitness training, the 60-minute main part was divided equally (20 minutes each) into endurance training on the bicycle ergometer, strength training with small equipment and flexibility training. In this training program, we intentionally kept the coordinative demands of the exercises as low as possible. 
Structural volume changes of the brain were quantified using magnetic resonance imaging (MRI). A Siemens MAGNETOM Verio 3 Tesla magnetic resonance imaging scanner was used to collect the imaging data for this study. Based on high-resolution T1-weighted MR images, the amygdala volumes were estimated separately for each hemisphere using the segmentation and registration tool FIRST, which is part of the FMRIB's Software Library (FSL) (Patenaude et al., 2011). Blood samples, which were always collected in the morning in a fasting state, were analyzed using an ELISA kit (Enzyme Linked Immuno Sorbent Assay) and tested for neutrophins (specifically BDNF). BDNF concentrations in blood plasma, blood serum, and whole blood were determined, as well as the ratio of blood serum to plasma BDNF concentrations. Long-term regular physical activity and moderate to intense exercise induce an increase in BDNF receptors in brain regions responsible for motor activity and prevent neurodegeneration (Erickson et al., 2011). To measure memory performance, the Verbal Learning and Memory Test (VLMT) was performed. This test measures the performance of verbal declarative episodic memory (Helmstaedter et al., 2001). Balance ability was assessed using the limits of stability (LoS) test. This measures numerous movement characteristics related to the subject's ability to swing voluntarily to different positions in space and briefly maintain balance at these positions. The test includes reaction time, movement speed, maximum deflection and endpoint deflection, as well as directional control. The Physical Working Capacity Test 130, a step load test on a bicycle ergometer, was used to evaluate the conditional capacity endurance. The performance parameters of the PWC 130 were absolute performance in watts and relative performance in watts per kilogram of body weight.

\section{Results}

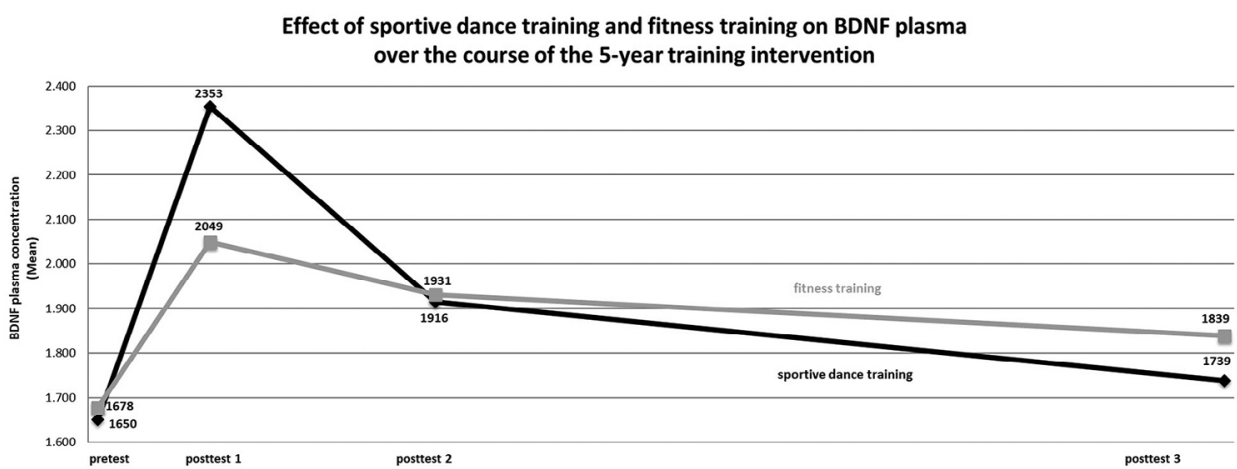

\section{Figure 1: Effect of sportive dance training and fitness training on BDNF plasma over the course of the 5-year training intervention}

The volume of the left amygdala increased significantly over the five-year training period $\left(\mathrm{F}(3,36)=3.760, \mathrm{p}=.019\right.$, partial $\left.\eta^{2}=.24\right)$. Cohen's effect size 
f was .56 , corresponding to a strong effect. According to post-hoc tests with Bonferroni correction, there was a trend toward an increase in volume in the left amygdala from posttest 1 to posttest $2(p=.085)$. There were no significant group differences. Furthermore, it was shown that BDNF concentration in blood plasma remained stable over the five-year training period $(\mathrm{F}(3,45)=$ 2.591, $\mathrm{p}=.064$, partial $\left.\eta^{2}=.15\right)$. No significant group differences were present.

In addition, it was revealed that the Corrected Recognition Performance in the VLMT was significantly improved over the five-year training period in the sportive dance group (Chi-Square $(3)=12.197, \mathrm{p}=.007, \mathrm{n}=8$ ) and remained stable in the fitness training group (Chi-Square(3) $=3.387, \mathrm{p}=.336, \mathrm{n}=9$ ). No significant group differences were found.

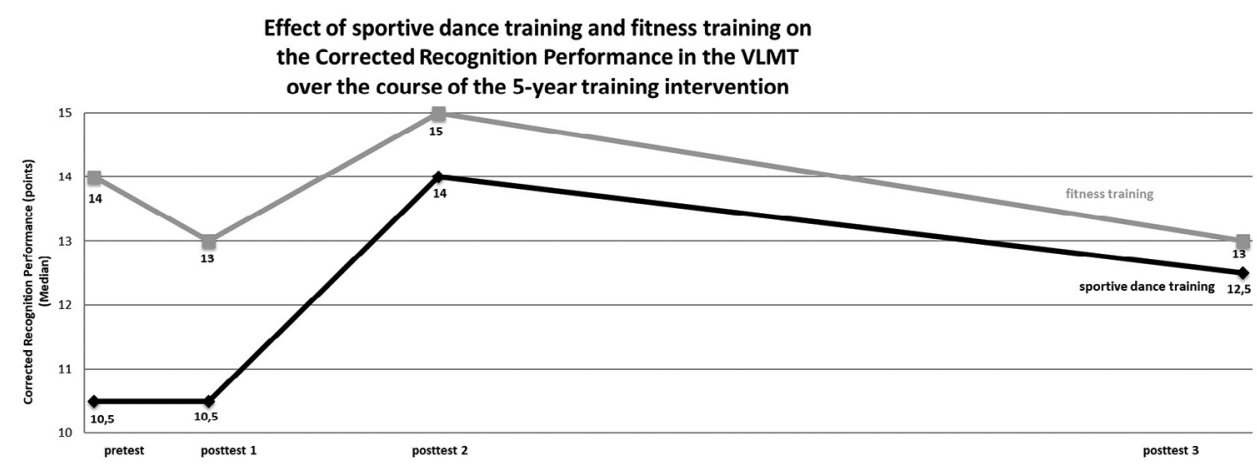

Figure 2: Effect of sportive dance training and fitness training on the Corrected Recognition Performance in the VLMT over the course of the 5-year training intervention

Moreover, it was shown that the "reaction time" in the LoS was significantly improved during the five-year training phase $(\mathrm{F}(3,42)=6.379, \mathrm{p}=.001$, partial $\eta^{2}=.31$ ). Cohen's effect size $\mathrm{f}$ is .67 , representing a strong effect. There were no significant group differences. The "speed of movement" in the LoS was also significantly increased during the five-year training period $(\mathrm{F}(3,42)=$ 4.925, $\mathrm{p}=.005$, partial $\eta^{2}=.26$ ). Cohen's effect size $\mathrm{f}$ was .59 , reflecting a strong effect. No significant group differences were observed. Furthermore, the "endpoint" in the LoS demonstrated a significant increase over the five-year training period $\left(\mathrm{F}(1.83,25.58)=9.221, \mathrm{p}=.001\right.$, partial $\left.\eta^{2}=.40\right)$. Cohen's effect size $\mathrm{f}$ was .81 , indicating a strong effect. There were no significant group differences. The "maximum deflection" in the LoS was also significantly enhanced $(\mathrm{F}(3,42)$ $=12.476$, $\mathrm{p}<.001$, partial $\eta^{2}=.47$ ). Cohen's effect size f was .94 , which corresponded to a strong effect. No significant group differences were present. 


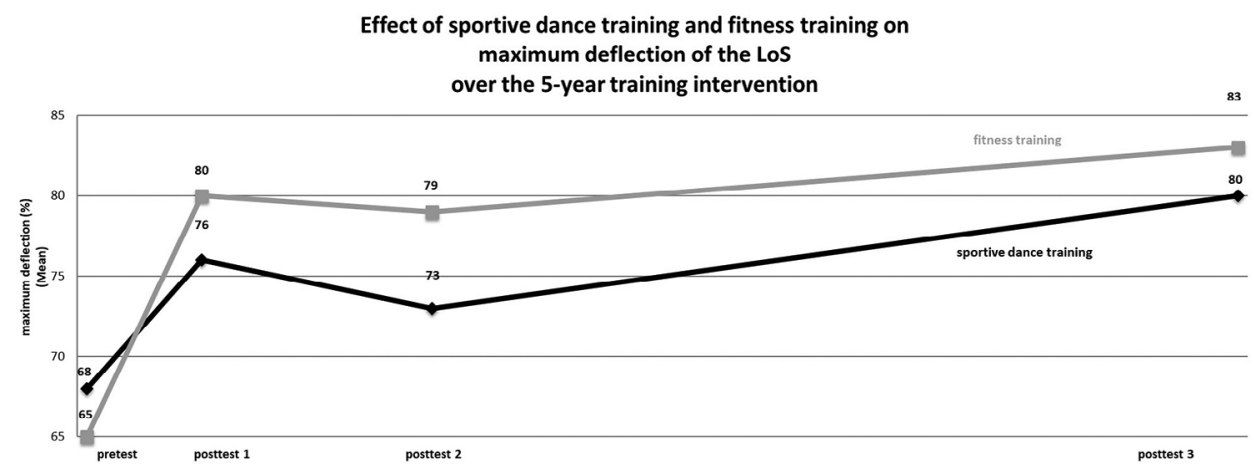

Figure 3: Effect of sportive dance training and fitness training on maximum deflection of the LoS over the 5-year training intervention

Finally, also with regard to "directional control" in the LoS, it could be shown that performance significantly increased from the second to the third measurement time point and significantly decreased again from the third to the fourth measurement time point $\left(\mathrm{F}(3,42)=6.542, \mathrm{p}=.001\right.$, partial $\left.\eta^{2}=.32\right)$. Cohen's effect size f was .68, corresponding to a strong effect. No significant group differences were present. Lastly, it was shown that absolute performance in watts of PWC 130 remained stable over the five-year training period in both the dance group (Chi-Square $(3)=4.846, \mathrm{p}=.183, \mathrm{n}=6$ ) and the fitness training group (Chi-Square $(3)=.360, \mathrm{p}=.948, \mathrm{n}=8$ ). There were no significant group differences. In addition, it was observed that relative performance in PWC 130 maintained stability $\left(\mathrm{F}(3,36)=1.189, \mathrm{p}=.328\right.$, partial $\left.\eta^{2}=.09\right)$. There have been no significant group differences. In both groups, conditional adaptations occurred in terms of stabilization of endurance performance over a 5 -year period.

\section{Discussion}

The present article reflects the main results of the longitudinal study. In the neurostructural and molecular area, a high heterogeneity of the results was observed. In the neuropsychological domain of the longitudinal study, there was a high homogeneity of results, which was characterized by a largely demonstrated maintenance of performance and an absence of group differences. An exception was the improvement of the corrected recognition performance in the sportive dance group. In the motor area of the longitudinal analysis, a maintenance of endurance performance and numerous improvements in balance ability were found.

\section{Conclusions}

Positive effects of sportive dance training as well as fitness training on neurostructural and molecular parameters in older adults during the five-year training intervention were found exclusively in left amygdala volume in both 
groups. Significant group differences were not detectable in the neurostructural and molecular domains. Beneficial effects on neuropsychological parameters were observed only in corrected recall (verbal memory) in the sportive dance group. Regarding motor parameters, positive effects on balance ability were demonstrated in both groups. Thus, it can be summarized that both the conditional-coordinative training dance and the purely conditional training of endurance and strength have a positive effect on neurostructural, neuropsychological as well as motor performance parameters in elderly people and can contribute to the preservation of motor and cognitive functions in old age. Therefore, a physically active lifestyle leads to maintaining quality of life and autonomy in old age.

\section{References}

ERICKSON, K. I., VOSS, M. W., PRAKASH, R. S., BASAK, C., SZABO, A., CHADDOCK, L., KIM, J. S., HEO, S., ALVES, H., WHITE, S. M., WOJCICKI, T. R., MAILEY, E., VIEIRA, V. J., MARTIN, S. A., PENCE, B. D., WOODS, J. A., MC AULEY, E. \& KRAMER, A. F. (2011). Exercise training increases size of hippocampus and improves memory. Proceedings of the National Academy of Sciences of the United States of America, 108 (7), 3017-3022.

FOLSTEIN, M. F., FOLSTEIN, S. E. \& MC HUGH, P. R., 1990. Mini-MentalStatus-Test. Weinheim: Beltz Test.

HELMSTAEDTER, C., LENDT, M. \& LUX, S., 2001. Verbaler Lern- und Merkfähigkeitstest. Göttingen: Beltz Test-GmbH.

MÜLLER, P., REHFELD, K., SCHMICKER, M., HÖKELMANN, A., DORDEVIC, M., LESSMANN, V., BRIGADSKI, T., KAUFMANN, J. \& MÜLLER, N. G. (2017). Evolution of neuroplasticity in response to physical activity in old age: the case for dancing. Frontiers in Aging Neuroscience, 9, 56.

PATENAUDE, B., SMITH, S.M., KENNEDY, D. \& JENKINSON, M. A. (2011). Bayesian Model of Shape and Appearance for Subcortical Brain Segmentation. NeuroImage, 56(3):907-922.

REHFELD, K., 2016. Zum Einfluss eines sportiven Tanztrainings und eines multimodalen Bewegungstrainings auf die Gehirnstruktur sowie auf kognitive und motorische Leistungen bei Senioren (Dissertation, Sportwissenschaft). Punkt um FILM, Biederitz.

REHFELD, K., LÜDERS, A., HÖKELMANN, A., LESSMANN, V., KAUFMANN, J., BRIGADSKI, T., MÜLLER, P. \& MÜLLER, N. G. (2018). Dance training is superior to repetitive physical exercise in inducing brain plasticity in the elderly. PLOS ONE, 13 (7), e0196636.

REHFELD, K., MÜLLER, P., AYE, N., SCHMICKER, M., DORDEVIC, M., KAUFMANN, J. HÖKELMANN, A. \& MÜLLER, N. G. (2017). Dancing or fitness sport? The effects of two training programs on hippocampal plasticity and balance abilities in healthy seniors. Frontiers in Human Neuroscience, 11, 305. 